\title{
Lateralized P3 deficit in schizotypal personality disorder
}

\section{Citation}

Niznikiewicz, Margaret A, Martina M Voglmaier, Martha E Shenton, Chandlee C Dickey, Larry J Seidman, EngKeat Teh, Richard Van Rhoads, and Robert W McCarley. 2000. "Lateralized P3 Deficit in Schizotypal Personality Disorder." Biological Psychiatry 48 (7) (October): 702-705. doi:10.1016/s0006-3223(00)00938-0.

\section{Published Version}

doi:10.1016/S0006-3223(00)00938-0

\section{Permanent link}

http://nrs.harvard.edu/urn-3:HUL.InstRepos:28520161

\section{Terms of Use}

This article was downloaded from Harvard University's DASH repository, and is made available under the terms and conditions applicable to Other Posted Material, as set forth at http:// nrs.harvard.edu/urn-3:HUL.InstRepos:dash.current.terms-of-use\#LAA

\section{Share Your Story}

The Harvard community has made this article openly available.

Please share how this access benefits you. Submit a story.

\section{Accessibility}


Published in final edited form as:

Biol Psychiatry. 2000 October 1; 48(7): 702-705.

\title{
Lateralized P3 Deficit in Schizotypal Personality Disorder
}

\author{
Margaret A. Niznikiewicz, Martina M. Voglmaier, Martha E. Shenton, Chandlee C. Dickey, \\ Larry J. Seidman, EngKeat Teh, Richard Van Rhoads, and Robert W. McCarley \\ From the Harvard Medical School Department of Psychiatry, Brockton/West Roxbury VAMC, \\ Brockton (MAN, MES, CCD, ET, RVR, RWM), the Harvard Medical School Department of \\ Psychiatry, Massachusetts Mental Health Center, Boston (MAN, LJS, ET, RVR), and the Harvard \\ Medical School Department of Psychiatry, Cambridge City Hospital, Cambridge (MMV), \\ Massachusetts
}

\section{Abstract}

Background-Reduced, left-lateralized P3 amplitude has been reported in several studies focusing on electrophysiologic function in schizophrenia. Also, several lines of evidence suggest a similarity between schizophrenia and schizotypal personality disorder (SPD). This study was undertaken to determine the replicability of our previous finding of a left-lateralized P3 amplitude deficit in SPD.

Methods-We recorded event-related potentials in 21 SPD and 18 normal control subjects in an auditory "oddball" P3 paradigm.

Results-In the SPD subjects, but not in the control subjects, there was lower P3 amplitude at T3 compared with T4.

Conclusions-These results are similar to the ones in our previous work and further support the presence of a left-lateralized P3 deficit in SPD.

\section{Keywords}

SPD; P300; schizophrenia; auditory oddball

\section{Introduction}

Schizotypal personality disorder (SPD) and schizophrenia may have a common genetic diathesis (Baron et al 1985; Kendler et al 1993), possibly leading to a neurocognitive outcome characterized by a similar, but less severe, dysfunction in SPD.

One of the more direct measures of neurocognitive function is event-related potentials (ERPs) and, in particular, the auditory $\mathrm{P} 3$, a positive-going potential appearing within 250 to $600 \mathrm{msec}$ after the target stimulus. It has been related functionally to memory updating (Donchin 1981), and its latency and amplitude have been related to stimulus evaluation (Kutas et al 1977). In particular, the $P 300$ left temporal amplitude reduction appears to be selective to schizophrenia relative to affective psychosis in both first episode and chronic subjects (Ford et al 1982; Salisbury et al 1998) and has been found in most (Faux et al 1988; Heidrich and Strik, 1997; Salisbury et al 1992, 1994; Strik et al 1994; Turetsky et al 1998; Weisbrod et al 1997) but not all (e.g., Pfefferbaum et al 1989; Stefansson et al 1996) studies of schizophrenia. 
Data suggest that smaller P3 amplitude is associated with volume reductions in the left posterior superior temporal gyrus (Seig et al 1991).

Given the genetic similarities between the two disorders, a P3 dysfunction, similar but less severe to that found in schizophrenia, might be expected in SPD. In fact, somewhat reduced (but not statistically significant) P3 amplitude was found in SPD when compared with normal control subjects (Kalus et al 1991; Squires-Wheeler et al 1997; Trestman et al 1995) and to borderline personality individuals (Kutcher et al 1987, 1989). Our previous study (Salisbury et al 1996), reported a left-lateralized P3 amplitude abnormality in a sample of 11 unmedicated SPD subjects and no differences at midline electrodes in 11 male subjects relative to 11 matched normal control subjects. In our present study, we used an identical ERP protocol in a new group of 19 unmedicated male SPD subjects, and 18 matched normal control subjects to evaluate the replicability of our previous findings in a new group of subjects. We predicted a smaller P3 amplitude in the SPD group relative to comparison subjects, especially over the left temporal area.

\section{Methods and Materials}

\section{Subjects}

Participating in the study were 21 right-handed individuals diagnosed with SPD using the SCID and DSM-III-R (diagnostic reliability between two raters (MV and LS, $\kappa=.87$ ), meeting full $(\geq 5)$ diagnostic criteria, and 18 male comparison subjects, screened with SCID, and matched for age and IQ. The SPD subjects responded to a newspaper advertisement calling for male, shy individuals with few friends who felt they had special powers. The 18 normal comparison men were recruited from an advertisement for an electroencephalogram (EEG) experiment. All subjects were paid and signed a written informed consent form before entering the study. English was the first language for all subjects.

The exclusion criteria for SPD and control subjects were 1) history of electroconvulsive treatment; 2) neurological illness; 3) history of traumatic brain injury with a loss of consciousness greater than $5 \mathrm{~min}$; 4) drugs or medications affecting cognitive function in the past year and any history of use or substance addiction, as defined by DSM-IIIR; 5) use of neuroleptics at any time; and, 6) hearing, vision, or upper body motor impairment. SPD subjects were allowed to have first-degree relatives with a history of psychiatric disease (Axis I disorder [s] were present in six of the SPD subject's first-degree relatives), whereas for normal control subjects, this clinical history was an exclusion criterion. The SPD subjects' average age was $37.78(23-53, \mathrm{SD}=10.41)$, the IQ was $111.96(80-139, \mathrm{SD}=14.71)$, parental socioeconomic status (SES) was $3.6(1-5, \mathrm{SD}=1.2)$. The comparison subjects' average age was $38.9(22-51$, $\mathrm{SD}=9.83$.), IQ was $116.8(109-135, \mathrm{SD}=14.3)$, and parental SES was $3.53(2-5, \mathrm{SD}=1.1$ ). There were no statistically significant group differences on any of the demographic measures, with the exception of subject SES (SPD, 3.1; comparison subjects, $4.1 ; p<.01$ ), which is not a matching variable because it may reflect the effects of the disorder.

\section{The P300 Protocol}

The P300 protocol was identical to that used by Bruder and associates (1992). A Neuroscan Stimulation program (Neuroscan, El Paso) generated 200 pure tones of $1.5 \mathrm{kHz}$ ( $15 \%$ of all tones) and $1 \mathrm{kHz}$ ( $85 \%$ of all tones), $40 \mathrm{msec}$ duration, $10 \mathrm{msec}$ rise/fall, $97 \mathrm{~dB}$ (the dB levels calibrated with $\mathrm{dB}$ meter), 1.2-sec interstimulus interval, were randomly presented to a subject via Etymotic insert earphones (Neuroscan). The subject was asked to silently count the number of high tones. Both groups were equally accurate in counting the number of target tones (95\% accuracy). 
An EEG was recorded during a cognitive task for $924 \mathrm{msec}$, with $100 \mathrm{msec}$ prestimulus baseline, sampled at the rate 256 with A/D rate of 250 . Linked mastoids were used as reference; caution was taken to keep both ears' impedance at below $5 \mathrm{k} \Omega$ and at no more than $.5 \mathrm{k} \Omega$ difference. The ERP to target (high tones) and nontarget (low tones) was averaged offline. Before averaging, the EEG was corrected for eye movements using Semlitsch (Semlitsch et al 1986) algorithm. Epochs containing voltage exceeding $\pm 75 \mathrm{mV}$ at any electrode location were rejected from further analysis. The averages were then filtered with a low-pass filter of $8 \mathrm{~Hz}$, $24 \mathrm{~dB} /$ octave rolloff to remove noise and alpha contamination from the data.

The P300 peak latency was measured within 320 to $420 \mathrm{msec}$ poststimulus latency window, and the amplitude was calculated as the mean integrated voltage under the curve within the same latency window. The amplitude and latency data were measured from three midline ( $\mathrm{Fz}$, $\mathrm{Cz}, \mathrm{Pz}$ ) and four temporal electrodes (T3/4 and T5/6) known to be sensitive to lateralized voltage abnormalities previously reported in schizophrenic and schizotypal subjects (McCarley et al 1993, Salisbury et al 1996). For P3 amplitude values at lateral electrodes, the P300 amplitude data were normalized using Cz voltage following McCarthy and Woods (1985) recommendations to remove the problems of variance misallocation before statistical analyses.

\section{Results}

The amplitude and latency data were submitted to repeated measures analyses of variance (ANOVAs) for two electrode chains (midline: Fz, Cz, Pz; and lateral: T3, T4, T5, T6), with group (two levels: SPD and control subjects) as a between variable and electrode as a within variable for midline (three levels: $\mathrm{Fz}, \mathrm{Cz}, \mathrm{Pz}$ ); for the lateral electrodes two within factors were analyzed, hemisphere (two levels: left and right) and electrode (two levels). The ANOVA for the midline electrode chain revealed the main effect of group $[F(1,35)=9.018, p<.005]$ with P3 amplitude in the SPD group smaller than in the normal control group (Figure 1). In the ANOVA for P3 amplitude at lateral electrode chain, no significant group $\times$ side interaction was found; however, given our prediction of abnormal amplitude distribution at lateral sites and the presence of unequal variances in the control and SPD groups (which may cause loss of power in ANOVA comparisons), we used unidirectional one-sample $t$ tests to examine amplitude distribution within each group for T3/4 and T5/6; the tests yielded a significant difference within the SPD group for T3/T5 $[t(20)=-1.804, p<.043]$ and a trend-level difference at T5/T6 $[t(18)=-1.676, p<.055$; Figure 2]. Lower amplitude was found at T3 (. $37, \mathrm{SD}=.6)$ relative to $\mathrm{T} 4(.57, \mathrm{SD}=.6)$, and at $\mathrm{T} 5(.50, \mathrm{SD}=.4)$ and $\mathrm{T} 6(.64, \mathrm{SD}=.45)$ in the SPD group. No difference in amplitude distribution at analogous electrode sites was found within the control group (T3 $.42, \mathrm{SD}=.2$ vs. T4 $.46, \mathrm{SD}=.27$; $\mathrm{T} 5.44, \mathrm{SD}=.2$ vs. T6 $.49, \mathrm{SD}$ $=.14$ ). No group differences were found in the P3 latency, measured at midline and lateral electrodes, although there was a trend toward a prolonged P3 latency $(p>.1)$ at midline in the SPD individuals.

\section{Discussion}

The aim of this study was to extend the findings of reduced, left-lateralized P3 amplitude in the SPD reported previously (Salisbury et al 1996) to a new group of subjects and thus better assess the generalizability of this finding to SPD population. Reduced P3 amplitude was found at midline electrodes (Figure 1), the finding different than that reported in previous studies where reduced P3 amplitude in SPD did not distinguish between normal and SPD subjects on statistical tests. It may be that this group difference is due to a slow positive wave that developed around P3 latency range and persisted for the rest of the epoch. At lateral electrodes, the results indicate that there was a small but significant reduction of $\mathrm{P} 3$ amplitude on the left side relative to the right side within the SPD group. Such reduction was not found in the normal control group. Importantly, the reduction was small enough not to be evinced in the group by 
hemisphere interaction at lateral temporal sides. The group differences consisted in the asymmetrical voltage distribution at T3 and its right-side homologue, T4, within the SPD group (Figure 2) and at a trend level at T5 and T6. In contrast, among normal control subjects, there was no statistical difference in the P3 amplitude distribution as a function of the left and right hemisphere. This finding is consistent with earlier reports of $\mathrm{P} 3$ abnormality in schizophrenic patients where it was suggested that an abnormality in structures of the left superior temporal gyrus selectively influences the generators contributing to the surface voltage at lateral sites, resulting in the left-side amplitude reduction. Because no such abnormality is expected in the normal subjects, no difference in lateral voltage distribution is expected. The results of this study suggest that a similar mechanism may be at work in the SPD sample. As has been noted, however, the impairment seems to be much more subtle; in this study, the laterality reversal was demonstrated only in within-group comparisons and not in direct hemisphere-by-site comparisons, as was the case with schizophrenic subjects. This is arguably a less strong result than a direct demonstration of a group-by-site interaction.

The differences in variance may be responsible. One of the factors influencing variability maybe the heterogeneity of SPD groups, where different degrees of impairment are found across samples. Also, the current results suggest that in terms of the electrophysiologic response, P3, the group separation between SPD and normal control subjects is smaller than between normal control and schizophrenic subjects, in agreement with their milder clinical presentation.

The study extends the findings reported in Salisbury et al (1996) in that a left-lateralized amplitude deficit and a latency prolongation were found in this new SPD sample. The results are different in that statistically significant separation in P3 voltage was found at midline electrodes and also by the fact that the statistical difference reported in Salisbury et al (1996) was at $\mathrm{T} 5 / 6$, and it was demonstrated in between-group rather than within-group comparisons. These differences underline the issue of the heterogeneity of the SPD population that may escape traditional sample comparisons, such as age, parental SES or education, all of which did not differ between the two groups.

The fact that paradigms used to probe $\mathrm{P} 3$ response in schizophrenia produce similar but less pronounced group differences between SPD and normal comparison subjects suggests that using the same paradigm to assess brain function across different clinical populations may be useful, as well as the fact that there may be a similarity of dysfunction in schizophrenia and SPD as measured by P3 oddball paradigm. Clinically, our results provide an additional piece of evidence for the common, probably genetically mediated, neurophysiologic link between schizophrenia, an Axis I disorder, and SPD, which is classified as an Axis II personality disorder.

In summary, the results of this study lend further support to the notion that SPD bears electrophysiologic features similar to those found in schizophrenia, although the lateral asymmetry group effect was attenuated in SPD. Further studies are needed to identify possible protective factors preventing individuals from developing schizophrenic symptoms. Also, further studies should focus on heterogeneity of the SPD group. We believe that both of these lines of inquiry might provide a better understanding of the clinical and neurophysiologic differences between SPD and schizophrenia.

\section{Acknowledgments}

This work was supported by the National Alliance for Research on Schizophrenia and Depression (MAN); National Institute of Mental Health Grants Nos. KO2-MH-0110 (MES), MH R29 50747 (MES), and MH R01 52807 (RWM); and the Department of Veterans Affairs Center for Basic and Clinical Neuroscience Research in Schizophrenia (RWM). 


\section{References}

Baron M, Gruen R, Rainer J, Kane J, Asnis L, Lord S. A family study of schizophrenic and normal control probands: Implications for the spectrum concept of schizophrenia. Am J Psychiatry 1985;142:447454. [PubMed: 3976917]

Bruder, G.; Towey, J.; Malaspina, D.; Gorman, J.; Tenke, C.; Kaufmann, C. Presented at the annual meeting of the APA; Washington, DC. 1992.

Donchin E. Surprise! ... Surprise? Psychophysiology 1981;18:493-513. [PubMed: 7280146]

Faux S, McCarley RW, Nestor P, Shenton M, Pollak S, Penhune V, et al. P300 topographic asymmetries are present in unmedicated schizophrenics. Electroencephalogr Clin Neurophysiol 1993;88:32-41. [PubMed: 7681389]

Faux S, Torello M, McCarley RW, Shenton M, Duffy F. P300 in schizophrenia: Confirmation and statistical validation of temporal region deficit in P300 topography. Biol Psychiatry 1988;23:776-790. [PubMed: 3365456]

Ford J, Duncan-Johnson C, Pfefferbaum A, Kopell B. Expectancy for events in old age: Stimulus sequence effects on P300 and reaction time. J Gerontol 1982;37:696-704. [PubMed: 7130643]

Heidrich A, Strik WK. Auditory P300 topography and neuropsychological test performance: Evidence for left hemispheric dysfunction in schizophrenia. Biol Psychiatry 1997;41:327-335. [PubMed: 9024956]

Kalus O, Horvath TB, Peterson A, Coccaro EF, Mitropoulou V, Davidson M, et al. Event related potentials in schizotypal personality disorder and schizophrenia. Biol Psychiatry 1991;29:137A.

Kendler K, McGuire M, Gruenberg A, O’Hare A, Spellman M, Walsh D. The Roscommon family study: I. Methods, diagnosis of probands and risk of schizophrenia in relatives. Arch Gen Psychiatry 1993;50:527-540. [PubMed: 8317947]

Kutas M, McCarthy G, Donchin E. Augmenting mental chronometry: The P300 as a measure of stimulus evaluation time. Science 1977;197:792-795. [PubMed: 887923]

Kutcher SP, Blackwood DHR, Clair DS, Gaskell DF, Muir WJ. Auditory P300 in borderline personality disorder and schizophrenia. J Gen Psychiatry 1987;44:645-650.

Kutcher SP, Blackwood DHR, Gaskell DF, Muir WJ, Clair DM. Auditory P300 does not differentiate borderline personality disorder from schizotypal personality disorder. Biol Psychiatry 1989;26:766774. [PubMed: 2590690]

McCarley RW, Shenton M, O’Donnell B, Faux S, Kikinis R, Nestor P, Jolesz F. Auditory P300 abnormalities and left posterior superior temporal gyrus reduction in schizophrenia. Arch Gen Psychiatry 1993;50:190-197. [PubMed: 8439239]

McCarthy G, Woods D. Scalp distributions associated with event-related potentials: An ambiguity associated with analysis of variance models. Electroencephalogr Clin Neurophysiol 1985;62:203208. [PubMed: 2581760]

Pfefferbaum A, Ford JM, White PM, Roth WT. P3 in schizophrenia is affected by stimulus modality, response requirements, medication status, and negative symptoms. Arch Gen Psychiatry 1989;46:1035-1044. [PubMed: 2573328]

Salisbury DF, O'Donnell BF, McCarley RW, Nestor PG, Faux S, Smith RS. Parametric manipulations of auditory stimuli differentially affect P3 amplitude in schizophrenics and controls. Psychophysiology 1994;31:29-36. [PubMed: 8146252]

Salisbury DF, O'Donnell BF, McCarley RW, Nestor PG, Smith RS, Haimson J. Stimulus discriminability effects on P3: Insensitivity of schizophrenics. Biol Psychiatry 1992;31:145A.

Salisbury DF, Voglmaier MM, Seidman LJ, McCarley RW. Topographic abnormalities of P3 in schizotypal personality disorder. Biol Psychiatry 1996;40:165-172. [PubMed: 8830949]

Seig K, Willsie D, Preston D, Gaffney G. Brain imaging: Evoked potential, quantitative EEG and SPECT abnormalities in schizophrenia. J Psychiatry Neurosci 1991;16:41-44. [PubMed: 2049369]

Semlitsch H, Anderer P, Schuster P, Presslich O. A solution for reliable and valid reduction of ocular artifacts applied to the P300 ERP. Psychophysiology 1986;23:695-703. [PubMed: 3823345]

Squires-Wheeler E, Friedman D, Amminger GP, Skodol A, Looser-Ott S, Roberts S, et al. Negative and positive dimensions of schizotypal personality disorder. J Personal Disord 1997;11:285-300. 
Stefansson SB, Jonsdottir TJ. Auditory event-related potentials, auditory digit span, and clinical symptoms in chronic schizophrenic men on neuroleptic medication. Biol Psychiatry 1996;40:19-27. [PubMed: 8780851]

Strik WK, Dierks T, Franzek E, Stober G, Maurer K. P300 asymmetries in schizophrenia revisited with reference-independent methods. Psychiatry Res 1994;55:153-166. [PubMed: 7870855]

Trestman RL, Keefe RSE, Mitropoulou V, Harvey PD, deVegvar ML, Lees-Roitman S, et al. Cognitive function and biological correlates of cognitive performance in schizotypal personality disorder. Psychiatry Res 1995;59:127-136. [PubMed: 8771227]

Turetsky BI, Colbath EA, Gur RE. P300 subcomponent abnormalities in schizophrenia: I. Physiological evidence for gender and subtype specific differences in regional pathology. Biol Psychiatry 1998;43:84-96. [PubMed: 9474441]

Weisbrod M, Winkler S, Maier S, Hill H. Left lateralized P300 amplitude deficit in schizophrenic patients depends on pitch disparity. Biol Psychiatry 1997;41:541-549. [PubMed: 9046986] 

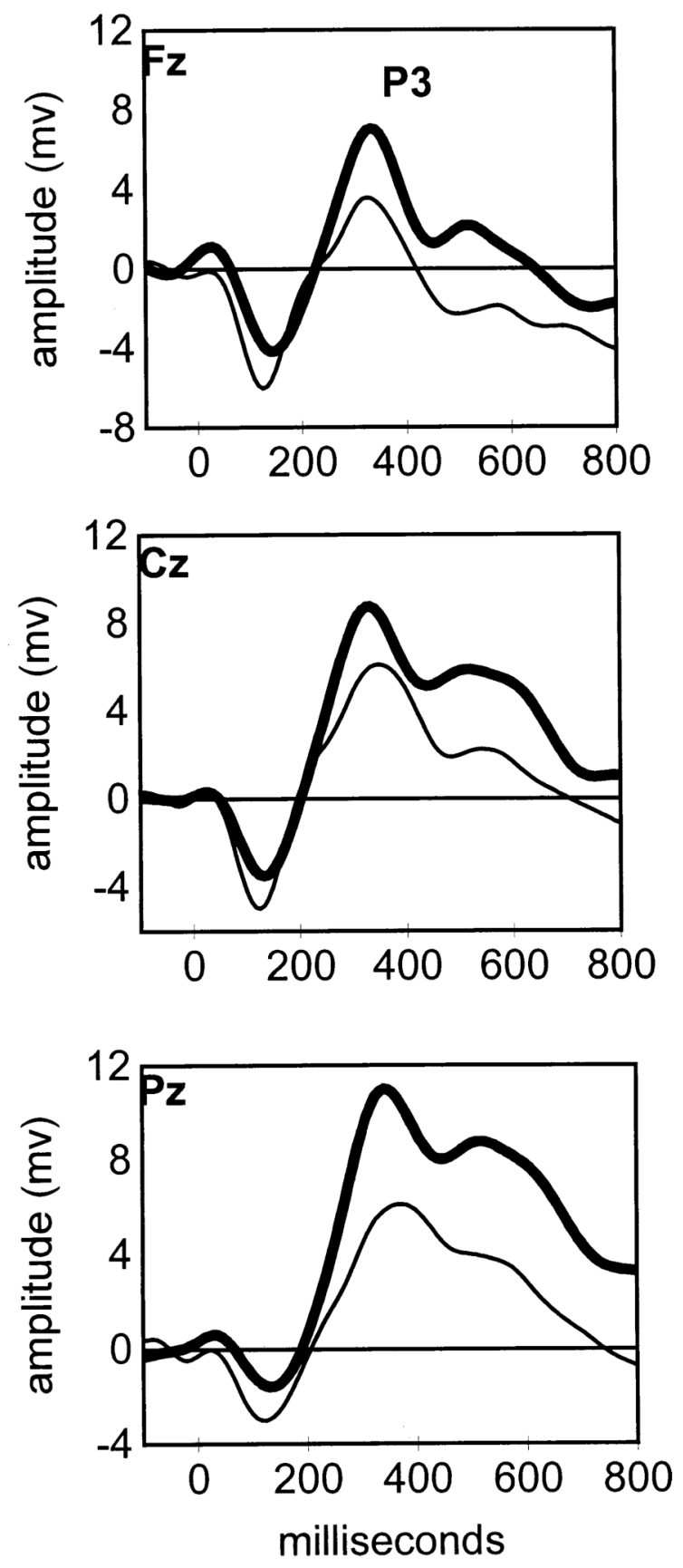

Figure 1.

Grand average waveforms of 18 normal control (NC; thicker line) subjects and 21 schizotypal personality disorder (SPD; thinner line) individuals to rare tones in the P300 auditory oddball paradigm at midline. 
T3

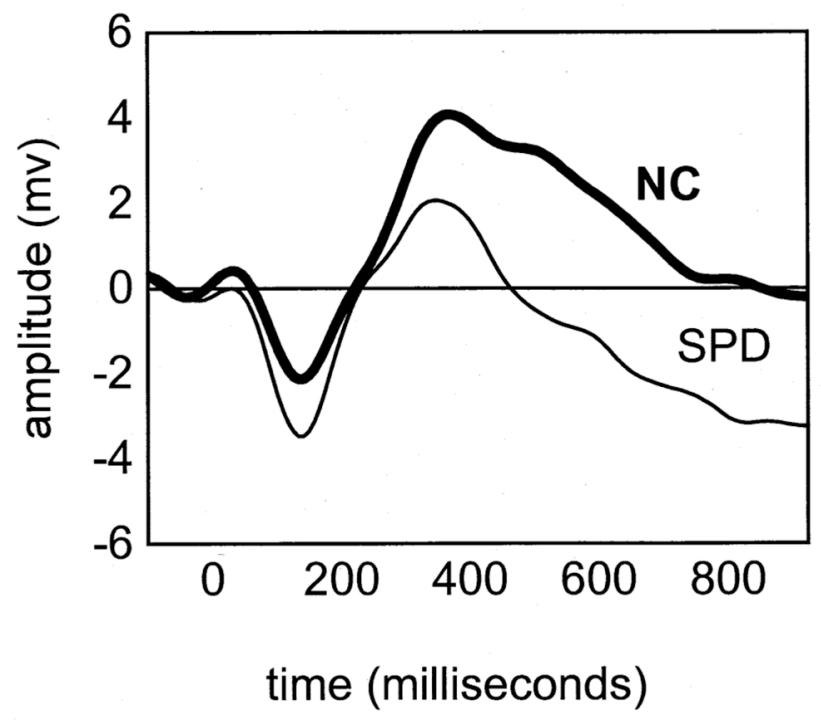

T4

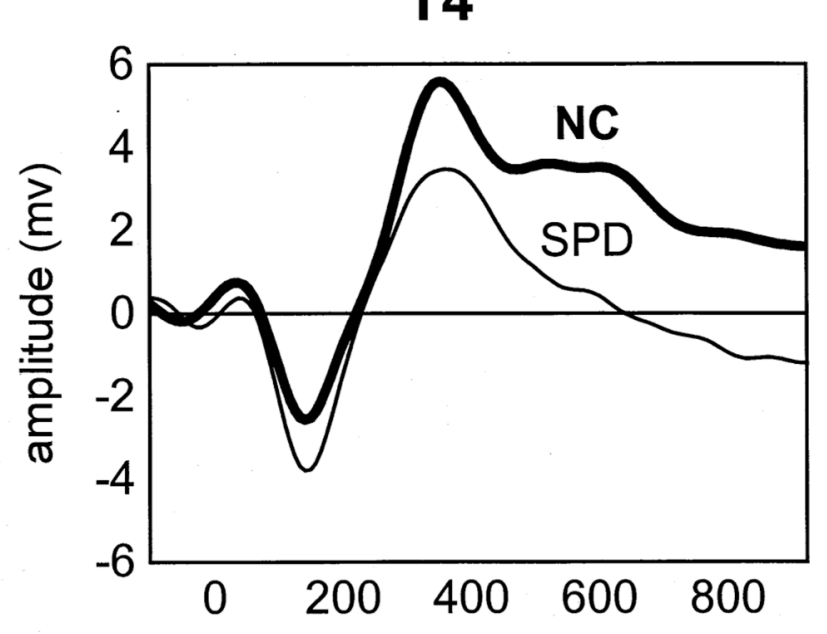

time (milliseconds)

Figure 2.

Grand average waveforms of 18 normal control (NC) subjects and 21 schizotypal personality disorder (SPD) individuals to rare tones in the P300 auditory oddball paradigm at lateral electrodes T3 and T4. 